\title{
Mesoscopic solute-rich clusters in olanzapine solutions
}

Monika Warzecha, † Mohammad S. Safari,‡ Alastair J. Florence, ${ }^{t, \S,{ }^{\star}}$ and Peter G. Vekilov¥, ll,*

†Strathclyde Institute of Pharmacy and Biomedical Sciences, University of Strathclyde, 161 Cathedral Street, Glasgow, G4 ORE, U.K.

‡Department of Chemical and Biomolecular Engineering, University of Houston, 4726 Calhoun Road, Houston, TX, 77204-4004, USA.

§EPSRC Future Continuous Manufacturing and Advanced Crystallisation Research Hub, University of Strathclyde, Technology and Innovation Centre, 99 George Street, Glasgow, G1 1RD, U.K.

"Department of Chemistry, University of Houston, 3585 Cullen Blvd., Houston, TX 77204-5003, USA

\section{ABSTRACT}

An organic molecule may crystalize in numerous distinct lattices and the slow and unpredictable transitions between multiple crystal forms is a significant concern in its pharmaceutical application. Recent results indicate that the transformation of olanzapine (OZPN) from anhydrous to hydrate crystals is mediated by mesoscopic solute-rich clusters. Here we demonstrate the existence of such clusters in undersaturated OZPN solutions in purely aqueous and mixed EtOH/aqueous solvents. The clusters occupy about $10^{-8}-10^{-7}$ of the solution volume and capture ca. $10^{-7}-10^{-5}$ of the dissolved OZPN. The average cluster radius is steady in time at about $35 \mathrm{~nm}$ and independent of the OZPN concentration and the solvent composition, whereas the OZPN fraction captured in the clusters is dictated by the solution thermodynamics. Both behaviors are in dire conflict with classical theories of phase transformation and recent aggregation models. They are, however, consistent with the predictions of a model that assumes the formation of OZPN dimers and their decay upon exiting the clusters. We propose that a 
transient dimer, which may be akin to the centrosymmetric dimer present in all of the 60 known OZPN crystal structures, may underlie cluster formation. The finding of cluster formation in organic systems and the proposed cluster mechanism provide guidance towards enhanced control over nucleation, molecular transitions, and the solid forms in molecular systems.

\section{INTRODUCTION}

Numerous organic and inorganic molecules exhibit multiple solid forms spanning polymorphs, solvates, salts, co-crystals, and amorphous structures. ${ }^{1-4}$ Distinct crystal forms can have different crystallization free energy and, correspondingly, different thermodynamic stability impacting key biopharmaceutical attributes including solubility. ${ }^{5}$ Even though the selection of the crystal form determines the fundamental properties and, ultimately, the utility of the crystals, the available understanding of polymorphism is mostly empirical. The Ostwald step rule, suggesting that less stable phases appear earlier, ${ }^{6}$ provides limited guidance on the direction of transitions between crystal forms. ${ }^{7}$ The mechanism of transition between solid forms, which dictates the kinetics of the process, is among the major unsolved problems of modern chemistry. ${ }^{8-9}$

Besides its fundamental significance, the potential variability of crystal forms has profound impact on the applications that rely of crystalline materials and their associated properties. ${ }^{10}$ A prime example is the manufacture of pharmaceutical products, where the majority of active pharmaceutical ingredients (APIs) are delivered as crystalline preparations and their bioavailability is governed by the crystal dissolution rate. ${ }^{11}$ As the crystal structure and composition dictate the solubility and bioavailability of the drug, polymorphic transformations are responsible for a wide range of formulation and manufacturing problems, such as unexpected 
formation of another form during production and storage ${ }^{12}$ or changes in the physical and chemical properties in gastric or intestinal environments. ${ }^{13-14}$
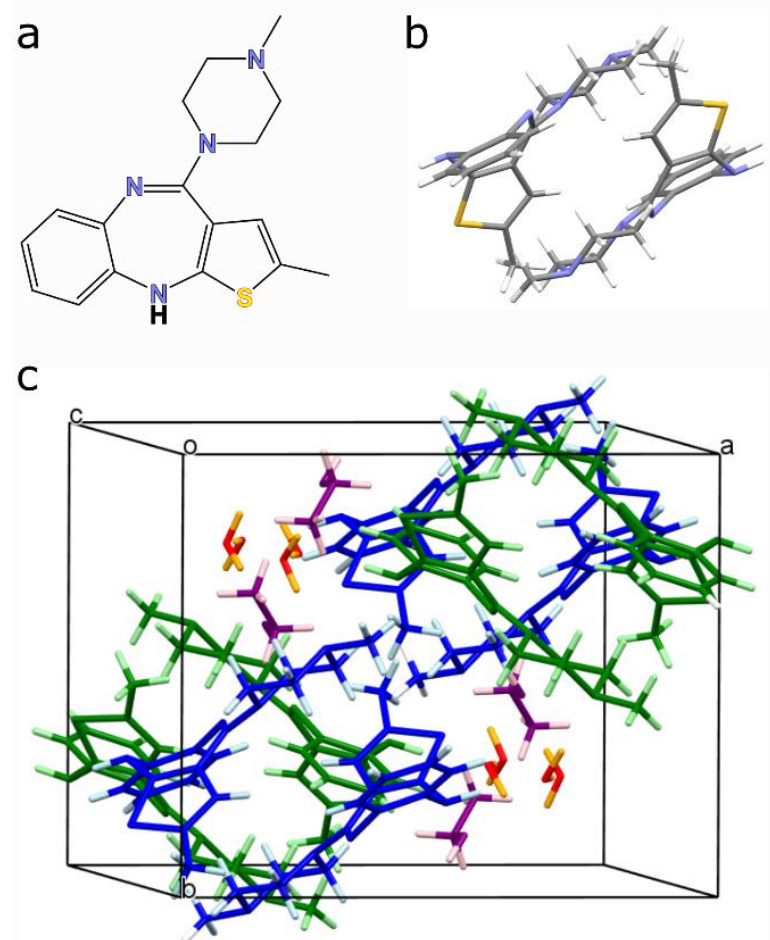

Scheme 1. The structure of olanzapine (OZPN). (a) The OZPN molecule. (b) The centrosymmetric dimer $(\mathrm{OZPN})_{2} \mathrm{SC}_{0}$ motif present in all crystal structures. ${ }^{15} \mathrm{~S}$, yellow; $\mathrm{N}$, blue; $\mathrm{C}$, are grey; and $\mathrm{H}$, white. (c) The crystal structure of the dihydrate ethanoate mixed solvate form OZPN:EtOH: $\mathrm{H}_{2} \mathrm{O}$ (2:1:2) in space group $P 2_{1} / \mathrm{c}$; Cambridge Structural Database REFCODE WEXQEW. OZPN dimers are drawn in in blue and green showing parallel dimers, EtOH molecules are drawn in purple, and water molecules, in red.

Recent results with the antipsychotic drug olanzapine (OZPN) ${ }^{16}$ Scheme 1a, have demonstrated that the transition between a non-solvated polymorph, OZPN I, and a dihydrate (OZPN DD) follows a mechanism distinct from the one envisioned by classical nucleation theory. To date, more than 60 individual forms of OZPN have been identified ${ }^{15}$ that include three non-solvated polymorphs, I, II, III. In aqueous solution, OZPN I transform to OZPN dihydrates, DB and DE, which are metastable, and the stable DD. These six forms exhibit very low aqueous solubility; however, exact values of the solubility have not been reported owing to the rapid 
phase transformation. ${ }^{14,}{ }^{17}$ In all solvated and neat forms, OZPN molecules are arranged as dimers, in which parallel plates are bound by reciprocal contacts between the thiophene methyl group and the piperazine entity of the paired molecule, Scheme $1 \mathrm{~b}$. In the solvates, such as the dihydrate-ethanoate depicted in Scheme 1c, the solvent molecules stabilize the packing by occupying the voids between the dimers. The dimer packing arrangement has been disturbed in a number of OZPN salts as a consequence of the introduction of competing interactions between counterions. ${ }^{18-19}$ Crystal structure prediction calculations ${ }^{15}$ have suggested that non-solvated crystal structures based on non-dimeric OZPN are thermodynamically feasible; however, none have been obtained to date.

The transformation of OZPN I to OZPN DD in unstirred solution is facilitated by an intermediate, liquid-like phase, that deposits as liquid clusters on $\{100\}$ faces of OZPN I, Figure 1a, and serves as nucleation medium for OZPN DD. ${ }^{20}$ When introduced into the solution bulk, the liquid clusters give rise to a metastable dihydrate polymorph, OZPN DB ${ }^{20}$ Both observations starkly diverge from the generally envisioned polymorph transition pathways. The obvious one, by structural rearrangement in the solid state, is more likely for crystals of isometric molecules or particles, interacting with a "soft" potential, in which the attractive minima are shallow, broad, and tolerant of molecular motion. ${ }^{21}$ For molecular crystals, in which the attractive forces are short-ranged, anisotropic, and strong, a more common option is by dissolution of the high freeenergy polymorph followed by crystallization of the stable form. 

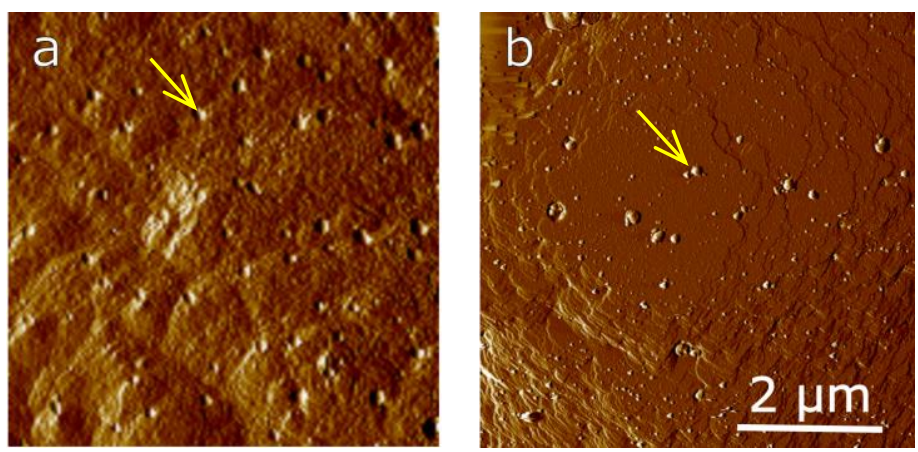

Figure 1. Dense OZPN-rich clusters (indicated with yellow arrows) on the surface of OZPN crystals imaged by atomic force microscopy. (a) Clusters on (100) face of OZPN I after incubation in water for 4 h. As demonstrated in Warzecha, et al, ${ }^{20}$, these clusters are a kinetic intermediate in the transformation from anhydrous OZPN I to the hydrated form OZPN DD. (b) Clusters on the surface of OZPN:EtOH: $\mathrm{H}_{2} \mathrm{O}$ after incubation for $1 \mathrm{~h}$ in saturated solution in $\mathrm{EtOH} / \mathrm{H}_{2} \mathrm{O} 1 / 1(\mathrm{v} / \mathrm{v})$.

Nucleation of OZPN DD and OZPN DB hosted by the dense liquid droplets contradicts the tenets of classical nucleation theory, according to which crystal embryos emerge in the solution by ordered assembly of solute molecules. ${ }^{22-25}$ On the other hand, it appears in accord with the two-step nucleation mechanism, according to which crystal nucleation is facilitated by dense liquid precursors. ${ }^{26-29}$ 30-31 This mechanism has been observed with protein, ${ }^{26-27,}$, 31-36 colloid, ${ }^{37-39}$ inorganic, ${ }^{40-42}$ and organic ${ }^{28,} 43-44$ molecules. Macroscopic dense liquid, appearing after "oiling out" and stable with respect to the solution, has been observed with organic molecules. ${ }^{45}$ The OZPN dense liquid clusters, however, do not represent a stable phase: they form a relatively monodisperse population with dimeter of ca. 80 nanometers and their growth upon deposition on the crystal surface appears to be not by association of monomers from the solution, but by coalescence with other clusters, Figure $1 .^{20}$ These clusters are also significantly larger than the disordered liquid clusters comprised of ca. 10 - 100 1,3,5-tris(4bromophenyl)benzene, or tribromide-Y molecules that host crystal nuclei; ${ }^{44}$ the size of the tribromide-Y clusters complies with the predictions of electrostatic aggregation models. ${ }^{46-47}$ 
Here we explore the enigma presented by the OZPN mesoscopic liquid clusters. We monitor the properties of the cluster population and its evolution and deduce the cluster formation mechanism. We draw an analogy to the protein-rich clusters of similar size, which assemble owing to the dynamics of formation and decay of transient dimers. ${ }^{48} \mathrm{We}$ compare the cluster behaviors in solvents that contain varying amount of ethanol and demonstrate that the fraction of OZPN, captured in the clusters, is dictated by the thermodynamic parameters of the solutions. On the other hand, the cluster size is decoupled form the volume of the cluster population and is likely determined by the dynamics of cluster assembly.

\section{MATERIALS AND METHODS}

Solutions. Analytical grade solvents were purchased from Fisher Scientific and Sigma Aldrich. OZPN was purchased from Molekula Ltd., UK and used without further purification. Weighted amount of OZPN were dissolved in water and water-ethanol mixtures. Prior to characterization of aggregation, all solutions were filtered through a $0.22 \mu \mathrm{m}$ PTFE filter.

Atomic Force Microscopy (AFM). AFM data were collected on a Dimension Fast Scan AFM (Bruker) equipped with Icon scanner, using PeakForce Tapping® mode at RT and ScanAsyst Air probe (Bruker) with nominal spring constant $k=0.4 \mathrm{~N} / \mathrm{m}$ and a nominal tip radius of $2 \mathrm{~nm}$. OZPN I crystals were mounted with epoxy glue to a cover slip, placed in a crystallization dish, and submerged in a $5 \mathrm{~cm}^{3}$ volume of deionized water; no solution flow was applied. All AFM data was analyzed using NanoScope Analysis 1.5 software (Bruker). Height images were corrected by first-order flattening.

Cluster characterization by oblique illumination microscopy (OIM). The method relies on light scattered at wavevectors of order $\mu \mathrm{m}^{-1}$ and probe lengthscales in the range $10^{-3}-$ 
$10 \mu \mathrm{m}$. The Rayleigh law, according to which the scattered intensity scales as the sixth power of the scatterers' sizes, makes this technique particularly well suited to study the mesoscopic clusters, which are 50- to $100 \mathrm{~nm}$ large, but are present at very low concentration. OIM is also referred to as Brownian microscopy ${ }^{30,49-50}$ or particle tracking. ${ }^{51}$ We use Nanosight LM10-HS microscope (Nanosight Ltd). A green laser $(532 \mathrm{~nm}$ ) illuminates a $500 \mu \mathrm{m}$ solution layer at an oblique angle such that the incident beam avoids the lens of a microscope positioned above the sample, Figure $2 a^{50,52}$ The light scattered by the clusters is seen as dark speckles in the negative image in Figure $2 \mathrm{~b}, \mathrm{c}$; as the clusters are smaller than the diffraction limit, the speckle size accounts for the deviation of the cluster position from the microscope focal plane. We recorded the Brownian trajectory of a cluster in the image plane, Figure $2 \mathrm{~d}$, and computed the correlation between the mean squared displacement $\left\langle\Delta x^{2}\right\rangle$ and the lag time $\Delta t$, Figure 2e. We determined its diffusion coefficient $D$ from the slope of the $\left\langle\Delta x^{2}\right\rangle(t)$ correlation; we used the first five data points as they provide the highest accuracy of $D .{ }^{53}$
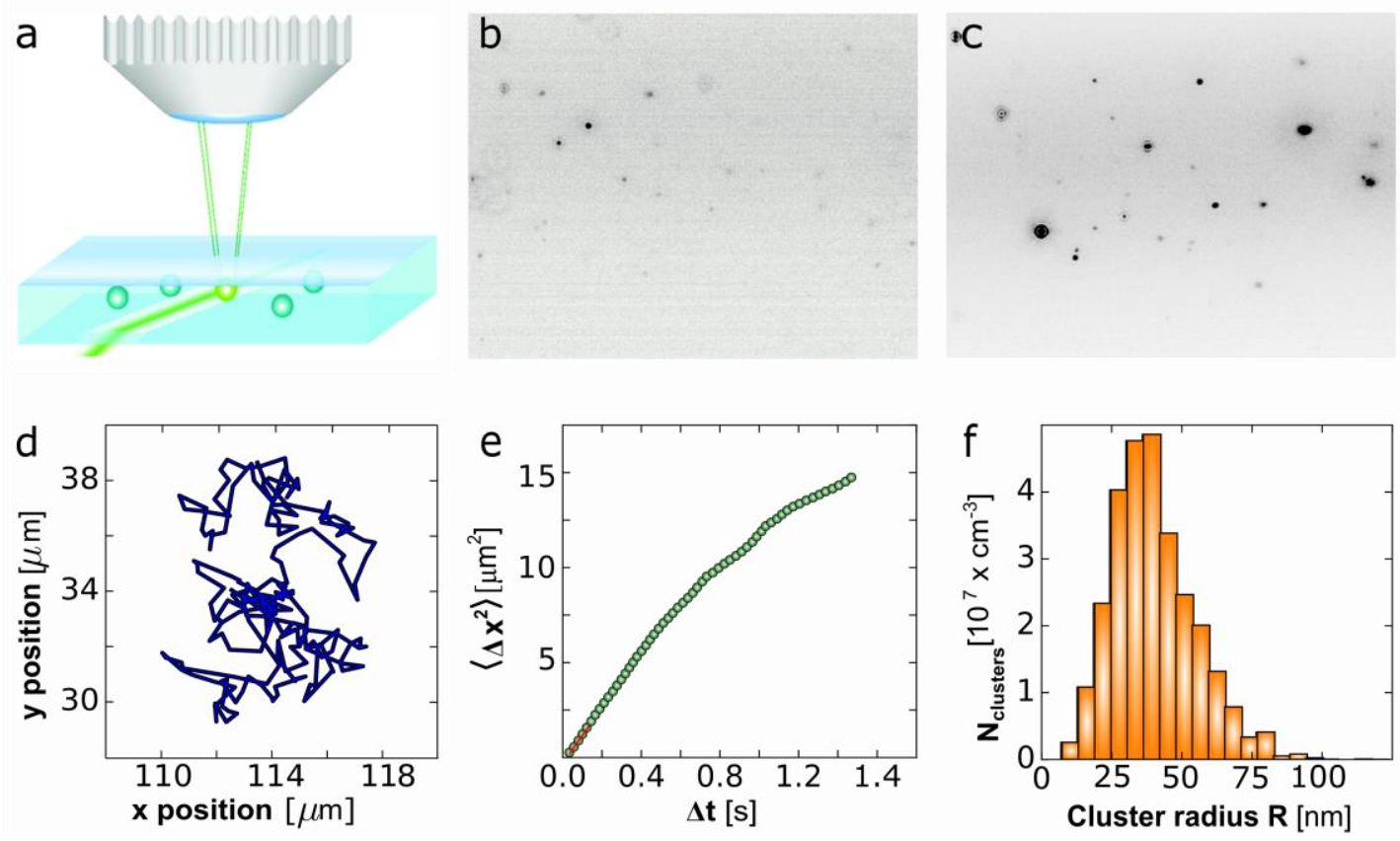
Figure 2. Characterization of the mesoscopic OZPN-rich clusters by oblique illumination microscopy (OIM). (a) The OIM set up. (b) A typical image, shown as a negative, in which the clusters appear as dark speckles, of OZPN clusters in $\mathrm{H}_{2} \mathrm{O}$. The observed volume is $120 \times 80 \times 5 \mu \mathrm{m}^{3}(\mathrm{~L} \times \mathrm{W} \times \mathrm{H})$. (c) A typical image of clusters in $3 \mathrm{mM}$ OZPN solution in EtOH/ $\mathrm{H}_{2} \mathrm{O} 80 / 20(\mathrm{v} / \mathrm{v})$. (d) A typical cluster trajectory obtained from the position of a cluster in a sequence of images. (e) Determination of the diffusion coefficient $D$ of a cluster from the correlation of its mean squared displacement $\left\langle\Delta x^{2}\right\rangle$ and the lag time $\Delta t$. First five data points are highlighted with a red line. (f) The distribution of cluster sizes obtained from five OIM movies recorded at distinct solution volumes.

We evaluated the cluster radius $R$ using the Stokes-Einstein relation, $R=k_{B} T / 6 \pi \eta D$. The temperature was set a $T=297.65 \mathrm{~K}$ and $k_{B}$ is the Boltzmann constant. $\eta$ is the viscosity of the solvent, determined from the dynamics of Fluoro-Max Dyed Red fluorescent polystyrene spheres, with diameter $1 \mu \mathrm{m}$, diffusing in the tested solutions. ${ }^{50,52}$ The viscosities of all solutions employed here are plotted in Figure S1. We evaluated the concentration $N_{\text {cluster }}$ of clusters of different sizes from the number $n$ in the monitored solution volume of $V=48,000 \mu \mathrm{m}^{3}, N_{\text {cluster }}=$ $n / V$, Figure $2 \mathrm{f}^{50,52-53}$ We evaluated the volume fraction occupied by the cluster population as $\phi=4 \pi\left(\sum R_{c}^{3} n_{c}\right) / 3 V$, where $V$ is the monitored solution volume. We repeated each characterization of the cluster populations five times from distinct solution volumes within the same sample and averaged $R, N_{\text {cluster }}$, and $\phi$ over the five measurements; the error bars in all plots represent the standard deviation of this determination.

\section{Determination of the optical extinction coefficient of OZPN in water-ethanol}

solvents. The ethanol concentration varied from 20 to $90 \% \mathrm{v} / \mathrm{v}$, with, correspondingly, 80 to 10 $\%$ water. Weighted OZPN amounts were dissolved in these solvents with concentrations between 0 and $40 \mu \mathrm{M}$. Completeness of dissolution was verified by the lack of scattered light using a device from ALV-GmbH, Langen, Germany. UV-Vis absorbance spectra were recorded, Figure S2. The wavelength of maximum absorbance varied between $252 \mathrm{~nm}$, for $20 \%$ ethanol, to 270 $\mathrm{nm}$, for $90 \%$ ethanol. The correlations between the optical density at the wavelength of 
maximum absorbance and the concentration were linear, Figure S2. The extinction coefficients $\varepsilon$ for each ethanol concentration were determined from the respective slopes and are summarized in Table S1.

\section{Determination of the temperature dependence of the OZPN solubility in water-}

ethanol solvents. We added aliquots of OZPN:EtOH: $\mathrm{H}_{2} \mathrm{O}(2: 1: 2)$ crystals to $5 \mathrm{~mL}$ solution with one of the tested compositions held in $20 \mathrm{~mL}$ capped glass vials. For experimental statistics, sets of three vials with identical composition were stored at each of five temperatures: $4,15,25,37$, and $45^{\circ} \mathrm{C}$. A $300 \mu \mathrm{L}$ aliquot was removed after preset time from each vial, diluted with the respective solvent, filtered through a $0.22 \mu \mathrm{m}$ PES filter, and the concentration of dissolved OZPN was determined spectrophotometrically. This procedure was repeated until the concentrations in each vial reached a plateau, defined by three consecutive concentrations of similar value, Figure S3. The final steady-state concentrations were averaged over the three samples for each temperature. The resulting mean was used as the solubility $C_{e}$.

X-ray Crystallography. XRPD patterns were obtained using a Bruker AXS D8-Advance transmission diffractometer equipped with $\theta / \theta$ geometry. The primary monochrome radiation was $\mathrm{Cu} \mathrm{K-} \alpha$, with wavelength $\lambda=1.54056 \AA$. Data were collected in the $2 \theta$ range $4-35^{\circ}$, with a $2 \theta$ step of $0.015^{\circ}$ and $8 \mathrm{~s} / \mathrm{step}$ speed. Reference powder patterns for the dihydrate ethanoate OZPN:EtOH: $\mathrm{H}_{2} \mathrm{O}$ (2:1:2) were computed using the Mercury 3.6 (CCDC) software from single crystal data from the Cambridge Structural Database. ${ }^{54}$

\section{RESULTS AND DISCUSSION}

Identification and reversibility of the mesoscopic OZPN-rich clusters. Undersaturated solutions of OZPN are expected to be homogeneous at all lengthscales, including the molecular. 
The extinction coefficient of OZPN in water is not available in the literature, and the low solubility in this solvent hampered its determination. Hence, to obtain an undersaturated OZPN solution we started with a solution saturated with respect to OZPN DB prepared by incubating overnight OZPN I crystals in water under constant stirring; under these conditions, OZPN I transforms to OZPN DB. ${ }^{20}$ We diluted this solution with $20 \%$ additional water by volume. The concentration of the diluted solution was below the solubility of OZPN DB. The most stable crystal form in aqueous solutions is OZPN DD; however, the transition to OZPN DD takes from five to seven days. ${ }^{20}$ Surprisingly, observations of the undersaturated solution with OIM revealed the presence of particles that randomly migrate, driven by Brownian collisions with the solvent molecules, Figure 2b. Careful examination of all steps in the solution preparation excluded the possibility that these heterogeneities are dust particles or gas bubbles. ${ }^{55}$ To test if the particles are crystals, we supplemented the IOM setup with a polarizer at the optical entrance of the cuvette and an analyzer at the optical exit, in front of the objective lens. If the polarizer and analyzer are in perpendicular orientation, crystals, which rotate the plane of light polarization, would produce bright speckles. Our tests revealed that the field of view was completely dark, disproving the hypothesis that the speckles seen without the polarizer in Figure $2 \mathrm{~b}$ represent crystals. We conclude that these particles are disordered.

We determined the size of the each particle from its Brownian trajectory. The average was ca. $35 \mathrm{~nm}$. This size is similar to the clusters observed on (100) surfaces OZPN crystals in aqueous solutions, Figure 1. Previous AFM observations of nucleation of new crystal polymorphs within the clusters ${ }^{20}$ indicate that the clusters are liquid. The cluster concentration in the pure aqueous solvent was low, likely owing to the low OZPN concentration, constrained by the poor solubility. This challenged further characterization of the clusters. As ethanol (EtOH) is 
known to significantly increase OZPN solubility, ${ }^{15}$ we prepared a solution with $C_{O Z P N}=3 \mathrm{mM}$ in $80 \%$ v/v EtOH. OIM characterization of this solution revealed a higher cluster concentration,

Figure 2c. The size distribution, Figure 2f, is relatively narrow, between 15 and $80 \mathrm{~nm}$, with a mean radius of ca. $35 \mathrm{~nm}$.
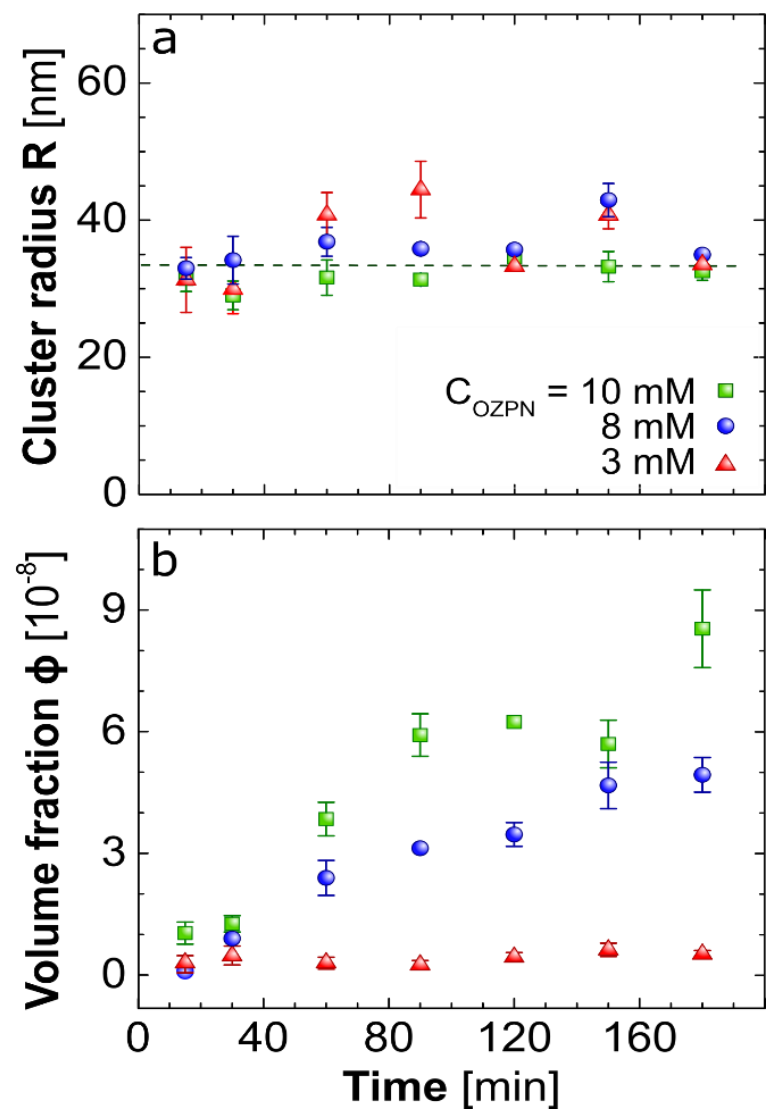

Figure 3. Evolution of the OZPN cluster population in $80 \% \mathrm{v} / \mathrm{v}$ EtOH solution. (a) The average cluster radius $R$. (b) The volume fraction $\phi$ in solutions with OZPN concentrations shown in (a). The averages of five determinations in distinct solution volumes are shown. The error bars represent the standard deviation.

To further characterize the mesoscopic OZPN-rich clusters, we monitored the time evolution of the cluster population and tested the consequences of varying $C_{O Z P N}$, Figure 3 . The cluster radius is independent of the solution concentration and steady in time for up to three hours, Figure 3a. In contrast, the fraction of the solution volume occupied by the cluster 
population $\phi$ increases with $C_{O Z P N}$ and solution age, Figure $3 \mathrm{~b}$. We verified that the evolution of $\phi$ is not due to OZPN precipitation or any other process in the solution hosting the clusters. The OZPN concentration, determined spectrophotometrically, remained steady. No changes in the solution UV-Vis spectra, illustrated in Figure S2, were recorded, suggesting that the $\phi$ growth may be induced by processes occurring in the liquid comprising the clusters, which stabilize the clusters and increase the cluster population by spawning additional clusters of consistent size. The slow growth of $\phi$ is consistent with slow maturation of the cluster phase. The high refractive index of this liquid, evidenced by the scattered intensity, suggests that the OZPN concentration in the clusters is higher than in the solution. The associated higher viscosity is conducive of slow dynamics.

A crucial issue in understanding the cluster mechanism is whether the clusters adjust to the parameters of the solution, or represent irreversibly aggregated matter. As a test of cluster reversibility, we note that the response of $\phi$ to higher $C_{O Z P N}$ is disproportional: as $C_{O Z P N}$ is raised by about three-fold, from 3 to $10 \mathrm{mM}, \phi$ increases from $0.5 \times 10^{-8}$ to $8.5 \times 10^{-8}$, i.e., ca. 17 -fold, Figure $3 b$; the latter ratio is consistent at all times, at which $\phi$ is sufficiently large for accurate distinction between the three concentrations. The exaggerated increase of $\phi$ driven by higher COZPN contradicts expectations for irreversible aggregates, whose concentration would increase proportionally to $C_{O Z P N}$. This increase indicates that the cluster population adjusts to the solution conditions and the clusters do not represent irreversibly aggregated material.

The characteristics of the cluster population revealed in Figures 2 and 3 are unusual for both disordered aggregates and emerging domains of a new phase, such as OZPN dense liquid. The narrow size distribution is inconsistent with both types of aggregation. The decoupled 
behavior of $R$ and $\phi$ are in contrast with classical phase transitions, in which the number of nucleated domains and the volume that they occupy increase concurrently. The mesoscopic size of the clusters is steady over extended times, in sharp contrast to expectations for newly formed phases in which the domain size grows in time. ${ }^{56}$ This size is much larger than the prediction of colloid clustering models ${ }^{46}$ that are often applied to aggregation in solution. ${ }^{47,57-63}$ As the cluster population captures a minor fraction of the dissolved OZPN, the concentration of the solution in contact with the clusters is close to the initial 3,8 , or $10 \mathrm{mM}$. This observation is beyond the catalogued behaviors of micelles, which equilibrate with solutions of constant critical micelle concentration (CMC). Thus, the OZPN clusters observed in Figures $1-3$ are not micelles.

On the other hand, these characteristics are typical of the mesoscopic solute-rich clusters, found in solutions of numerous proteins at varying conditions. ${ }^{35,48,64-67}$ Similar to OZPN clusters, the protein cluster size is steady and independent of the parameters that define the solution thermodynamics, such as $\mathrm{pH}$, ionic strength, and protein concentration. ${ }^{52,65-66,68} \mathrm{By}$ contrast, the volume fraction of the clusters is determined by the solute intermolecular interactions. ${ }^{30,48,52,65}$ A recent theory explained a range of puzzling cluster behaviors; ${ }^{48,}$ 69-70 several of the model assumptions and resulting predictions have been supported by experimental evidence $^{30,52,71}$ This model posits that the mesoscopic clusters consist of a concentrated mixture of intact monomers and transient dimers. The dimer lifetime determines the cluster radius $R$. On the other hand, in agreement the experimental data, ${ }^{30,48,52,65}$ the cluster volume fraction $\phi$ is dictated by the solution thermodynamics. ${ }^{48,52} \phi$ is related to the solute fraction $v$ held in the clusters. Indeed, $v=n_{c l} / n_{O Z P N}=C_{c l} V_{\mathrm{cl}} / C_{O Z P N} V=\left(C_{c l} / C_{O Z P N}\right) \phi$, where $n_{c l}$ and $n_{O Z P N}$ are the amounts of OZPN in the clusters and solution, respectively, $V_{\mathrm{cl}}$ and $V$ are the corresponding volumes, and $\phi=V_{\mathrm{cl}} / V{ }^{65}$ The OZPN concentration in the dense liquid comprising the clusters, 
$C_{c l}$, is significantly greater than $C_{O Z P N}$, so that choosing a constant ratio $C_{c l} / C_{O Z P N}$ in the range 10 -100 is reasonable. Furthermore, direct application of the Boltzmann distribution suggests that $v$ $\approx \exp \left(-\Delta G_{c l} / k_{B} T\right)$, where $\Delta G_{c l}$ is the free energy excess of a molecule in a clusters over that in the solution. ${ }^{48}$ Thus, we obtain that $\phi$ is determined by the free energy balance of clusters formation and the decoupled behaviors of $R$ and $\phi$ emerge naturally from the model ${ }^{48}$

The response of the cluster population to varying EtOH concentration. To test the applicability of the transient dimer model to the OZPN clusters, we monitored the response of the cluster population to varying concentration of $\mathrm{EtOH}$. The cluster radius $R$ is independent of the EtOH concentration in the range $20-90 \%$, Figure 4a. On the other hand, $\phi$ depends on $C_{E t O H}$ in a complicated non-monotonic fashion, Figure $4 \mathrm{~b}$. Concurrently with observations of mesoscopic clusters in protein solutions and in compliance with the predictions of the transient dimer model, $R$ and $\phi$ are decoupled. 

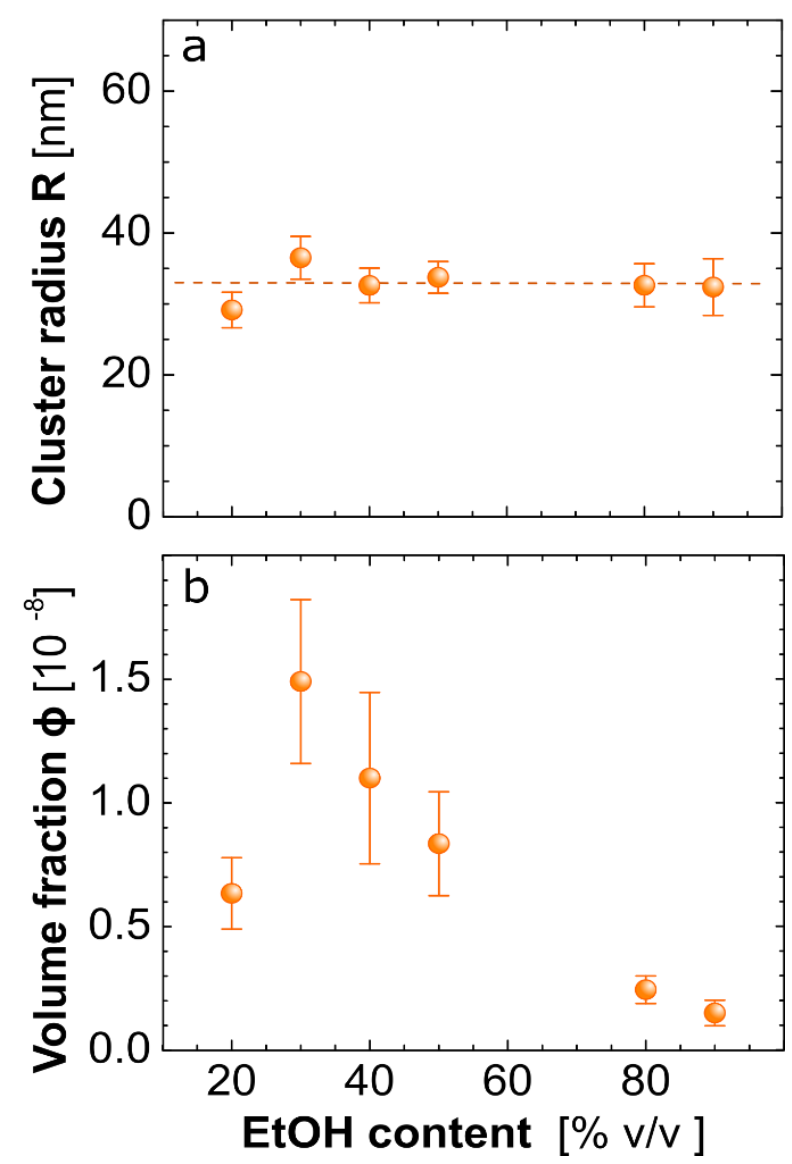

Figure 4. The cluster population in $\mathrm{EtOH} / \mathrm{H}_{2} \mathrm{O}$ solvents of different composition. (a) The average cluster radius R. (b) The volume fraction occupied by the cluster population $\varphi$ on EtOH concentration. The OZPN concentration was $5 \mathrm{mM}$ in all experiments. The averages of five determinations in distinct solution volumes are shown. The error bars represent the standard deviation.

\section{The thermodynamics parameters of OZPN crystallization from EtOH/water}

mixtures. To understand if the variations of $\phi$ in response to increasing $C_{E t O H}$ are driven by the thermodynamic parameters of the solution, we characterize the thermodynamics of OZPN solutions in mixed EtOH/water solvents. We choose the OZPN crystals as a reference state. Xray powder diffraction spectra of crystals grown at different EtOH/water ratios (Figure 5) reveal that OZPN crystallizes from all solvents as the OZPN dihydrate ethanoate, depicted in Scheme 1c. The crystallization enthalpy $\Delta H_{c r y s t}^{o}$, entropy $\Delta S_{\text {cryst }}^{o}$, and free energy $\Delta G_{\text {cryst }}^{o}$ characterize the difference between the crystals and the solution. Since the crystals grown in all tested 
solvents belong to the same polymorphic form, the disparities of $\Delta H_{c r y s t}^{o}, \Delta S_{\text {cryst }}^{o}$, and $\Delta G_{\text {cryst }}^{o}$ in different solvents distinguish the state of the OZPN solute in each solvent.

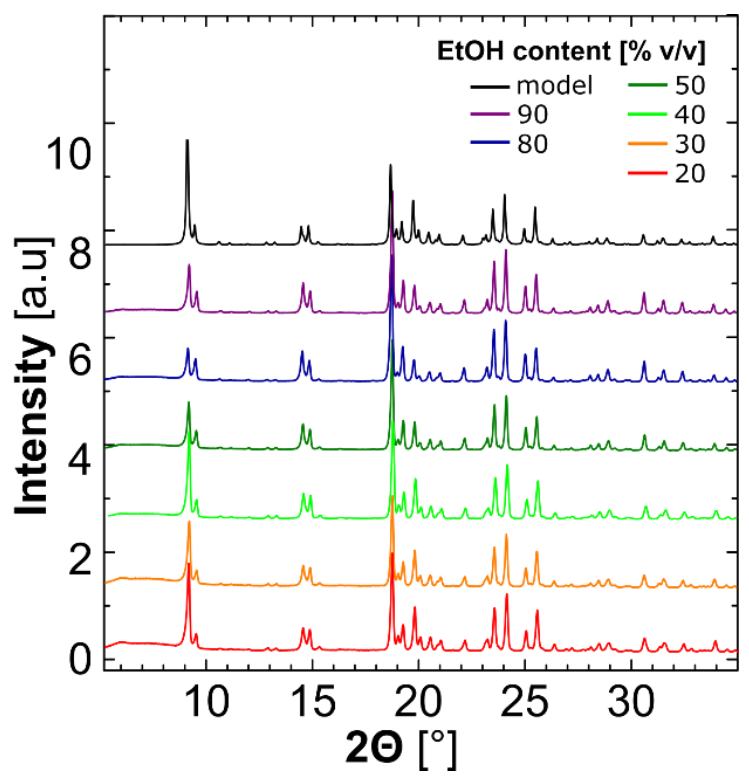

Figure 5. X-ray powder diffraction patterns of crystals grown in EtOH/H2O solvents with composition listed in the legend. Top: a model pattern computed using the software package Mercury and structure coordinates for the crystals of OZPN dihydrate ethanoate with Cambridge Structural Database entry WEXQEW.

The crystallization enthalpy $\Delta H_{\text {cryst }}^{o}$ can be measured calorimetrically by scaling the heat released during crystallization (at constant temperature and pressure $p$ ) with the crystallized mass. This determination can be distorted by solution trapped between crystals and mislabeled as crystalline mass. An alternative method is to determine the solubility $C_{e}$ of the crystals at different temperatures and to employ standard thermodynamics relations and $C_{e}(T)$ to determine $\Delta G_{\text {cryst }}^{o}, \Delta H_{\text {cryst }}^{o}$, and $\Delta S_{\text {cryst }}^{o} .^{72-73}$ 

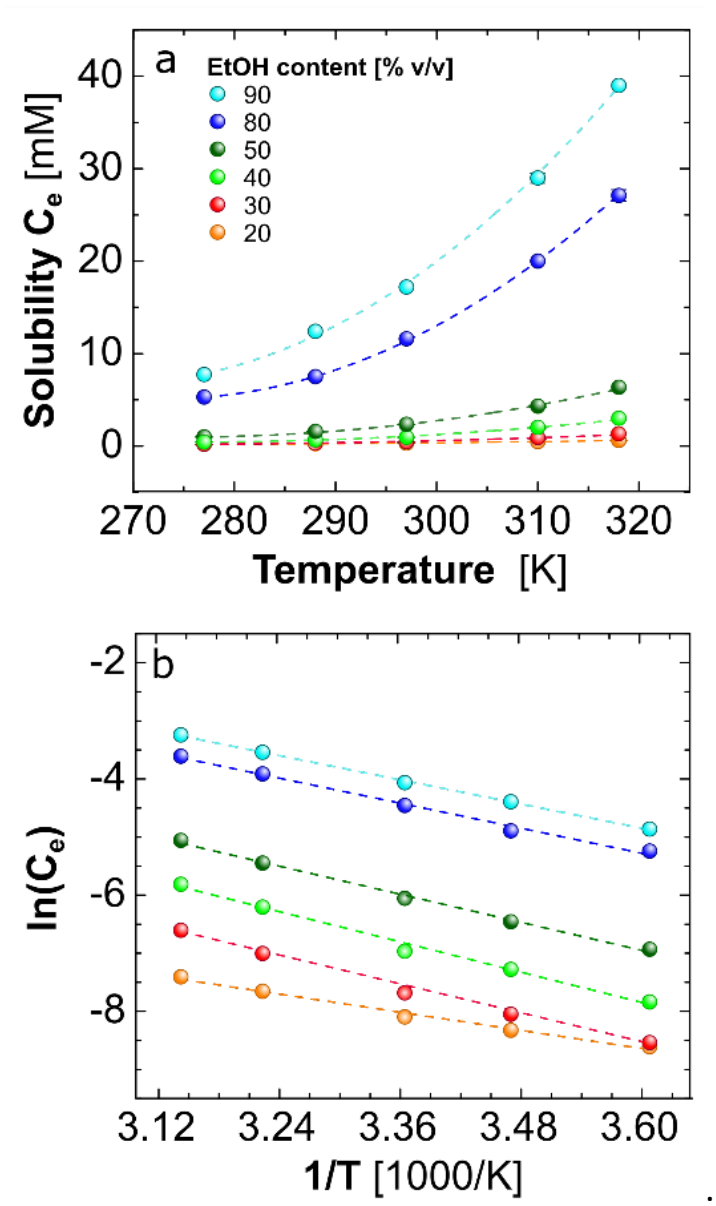

Figure 6. The solubility of OZPN with respect to the crystals of OZPN ethanol hydrate in $\mathrm{EtOH} / \mathrm{H}_{2} \mathrm{O}$ solvents of composition shown the legend in (a). (a) The temperature dependence of the solubility $C_{e}$ in the range $4-45^{\circ} \mathrm{C}$, dashed lines are polynomial fits. The standard deviation of three independent determinations carried out in parallel is smaller than the symbol size. (d) OZPN solubility plotted in van 't Hoff coordinates, dashed lines are linear regression fits.

The solubilities of OZPN with respect to the OZPN dihydrate ethanoate crystals in six $C_{E t O H}$ follow the expected quasi-exponential dependence on temperature, Figure 6a. The equilibrium constant for the reaction OZPN(solution) $\leftrightarrows \mathrm{OZPN}\left(\right.$ crystals) is $K=C_{e}^{-1}$. We use that the product, OZPN(crystals), represents a distinct phase and its activity is assigned to be one by thermodynamic convention. Furthermore, owing to the low $C_{e}$, we assume that the respective activity coefficients are close to one. Hence, $\Delta G_{c r y s t}^{o}=-R T \ln K=R T \ln C_{e}$. To determine $\Delta H_{\text {cryst }}^{o}$, we use the van ' $t$ Hoff relation written as 


$$
\left[\frac{\partial \ln C_{e}}{\partial(1 / T)}\right]_{p, n_{j}}=\frac{\Delta H_{c r y s t}^{0}}{R}
$$

where $n_{j}$ designates the concentrations of the other solution components, EtOH and water. The van ' $\mathrm{t}$ Hoff relation suggests that the slope of the correlation $\ln C_{e}\left(T^{-1}\right)$ is proportional to $\Delta H_{\text {cryst }}^{o}$. The data on OZPN solubility, plotted in van ' $t$ Hoff coordinates, Figure $6 \mathrm{~b}$, indicate that $\Delta H_{\text {cryst }}^{o}$ is constant in the studied temperature range. Finally, $\Delta S_{\text {cryst }}^{o}=\left(\Delta H_{c r y s t}^{o}-\Delta G_{c r y s t}^{o}\right) / T$ and is proportional to the intercept of the $\ln C_{e}\left(T^{-1}\right)$ plots, Figure $6 \mathrm{~b}$.

$\Delta S_{\text {cryst }}^{o}$ is a sensitive probe of the molecular-level processes that accompany crystallization. An OZPN molecule in the solution is surrounded by solvent molecules, some of which may be associated with OZPN. ${ }^{74-76}$ For instance, water molecules exposed to the nonpolar OZPN moieties would form a structured shell, surrendering their mobility. ${ }^{77}$ Recent modeling suggests that five water molecules are tightly associated with each OZPN in the solution and are positioned in a way that preserves their bond when dimers form. ${ }^{20}$ When an OZPN molecule incorporates in the crystal, some of the associated solvent molecules may be released and in this way regain their translational and rotational degrees of freedom. $\Delta S_{\text {cryst }}^{o}$ has a negative component due to the lost degrees of freedom as one OZPN, one EtOH, and two water molecules are confined to the crystal lattice. A positive component accounts for gained freedom of solvent molecules attached to OZPN in the solution and released upon crystallization. $\Delta S_{c r y s t}^{o}$ represents the balance between the two. The entropy loss corresponding to the incorporation of water molecules in ice is ca. $-22 \mathrm{~J} \mathrm{~mol}^{-1} \mathrm{~K}^{-1}$, consistent for water entrapped in clathrates, crystal hydrates, or other ice-like structures. ${ }^{78-82}$ For the larger EtOH, the corresponding number, evaluated from the entropy of fusion, is $-31 \mathrm{~J} \mathrm{~mol}^{-1} \mathrm{~K}^{-1} .83$ The relevant parameter for OZPN would be the entropy of crystallization from the melt, which is not available in the literature. As 
OZPN is significantly larger than EtOH, the entropy lost upon entrapment should have greater magnitude than $-31 \mathrm{~J} \mathrm{~mol}^{-1} \mathrm{~K}^{-1}$.
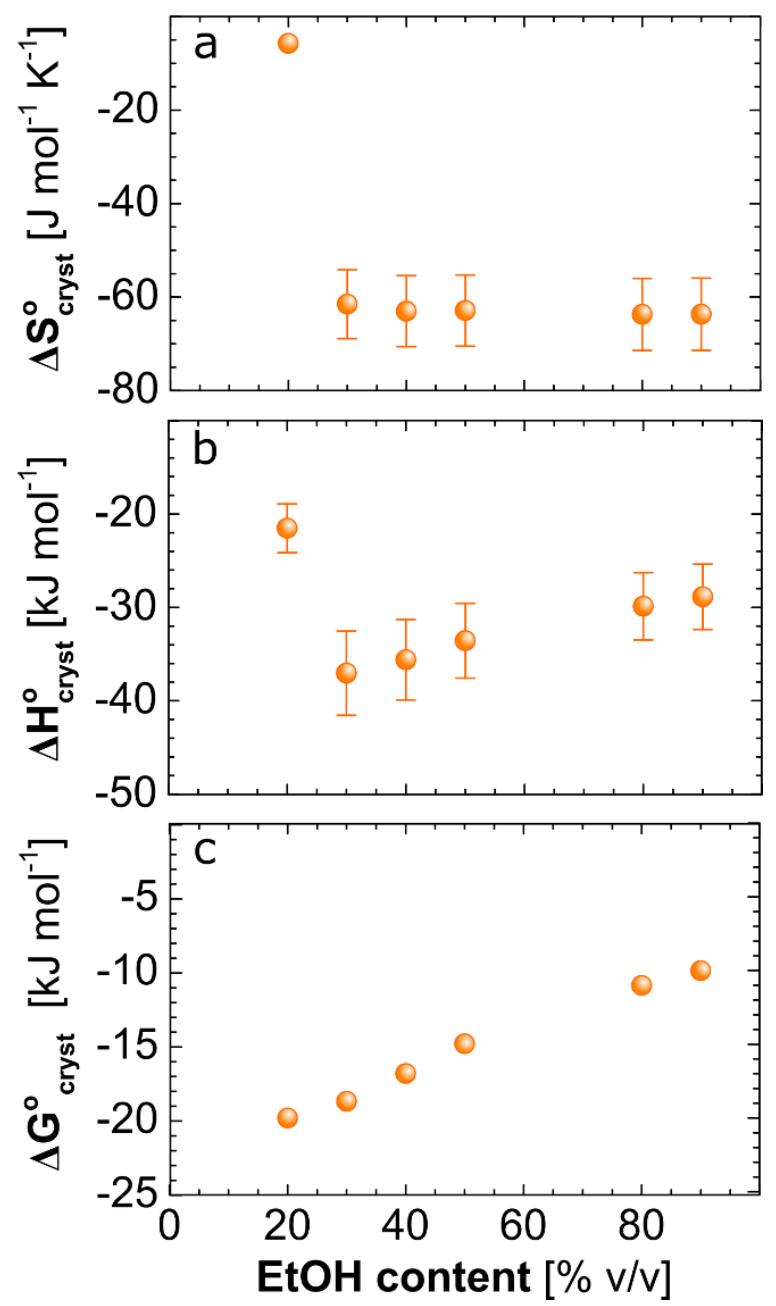

Figure 7. The thermodynamic parameters of crystallization of OZPN ethanol hydrate in $\mathrm{EtOH} / \mathrm{H}_{2} \mathrm{O}$ solvents of different. (a) The crystallization entropy $\Delta S_{\text {cryst }}^{o}$. (b) The crystallization enthalpy $\Delta H_{\text {cryst }}^{o}$. (c) The Gibbs free energy $\Delta G_{\text {cryst }}^{o}$ at $298 \mathrm{~K}$. The error bars in (a) and (b) represent the standard deviation of the intercept and slope, respectively, of the linear correlations in Figure 6b. The standard deviation for $\Delta G^{\circ}$ was determined form that of $C_{e}$ and is smaller than the symbol size.

The dependence of $\Delta S_{\text {cryst }}^{o}$ on the EtOH content in the solvent transitions sharply from 3 to $-62 \mathrm{~J} \mathrm{~mol}^{-1} \mathrm{~K}^{-1}$ as the $C_{E t O H}$ increases from 20 to $30 \% \mathrm{v} / \mathrm{v}$ and stays constant for $C_{E t O H}$ between 30 and $90 \% \mathrm{v} / \mathrm{v}$, Figure $7 \mathrm{a}$. The values of $\Delta S_{\text {cryst }}^{o}$ at both sides of the transition result 
from the balance of confined OZPN, EtOH, and water and released solvent molecules and cannot be interpreted in the absence of data on the structure of the solute - solvent complexes. The magnitude of the transition, however, ca. $60 \mathrm{~J} \mathrm{~mol}^{-1} \mathrm{~K}^{-1}$, suggests that three water molecules may be associated with OZPN in the $20 \% \mathrm{EtOH}$ solution and set free upon incorporation of OZPN into the crystal. This number is consistent with the five waters predicted to tightly bind to $\mathrm{OZPN}^{20}$ and the two water molecules trapped in the lattice. In solutions with EtOH content of 30 $\% \mathrm{v} / \mathrm{v}$ and higher, these water molecules are displaced from the vicinity of OZPN and do not affect the entropy balance of crystallization. The constant value of $\Delta S_{\text {cryst }}^{o}$ in the EtOH range from 30 to $90 \% \mathrm{v} / \mathrm{v}$ suggest that no additional water molecules are dissociated from solute OZPN by higher EtOH concentrations.

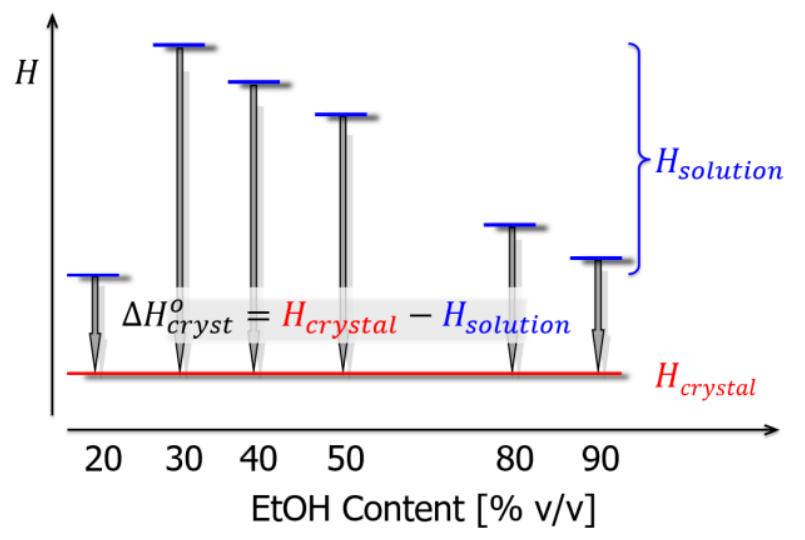

Scheme 2. The enthalpies of the solution and crystal emerging from the $\Delta H_{\text {cryst }}^{o}$ data in Figure $7 \mathrm{~b}$. The arrows indicate the transition from solution to crystals and their negative direction corresponds to the sign of $\Delta H_{c r y s t}^{o} . H_{\text {solution }}$ varies with the EtOH content antisymmetrically to $\Delta H_{c r y s t}^{o}$.

$\Delta H_{c r y s t}^{o}$ denotes the difference of the enthalpies of OZPN in the crystal and solution, $\Delta H_{\text {cryst }}^{o}=H_{\text {crystal }}-H_{\text {solution }}$. Since the crystals forming at different $C_{E t O H}$ are identical, $H_{\text {crystal }}$ does not depend on the $\mathrm{EtOH}$ content and the variations of $\Delta H_{\text {cryst }}^{o}$ are determined by $H_{\text {solution }}$, Scheme 2. $H_{\text {solution }}$ is a measure of solution non-ideality due to intermolecular 
attraction and repulsion, with higher $H_{\text {solution }}$ implying stronger repulsion. ${ }^{84}$ Thus, the low magnitude of $\Delta H_{\text {cryst }}^{o}$ at $20 \% \mathrm{EtOH}$, Figure $7 \mathrm{~b}$, suggests small $H_{\text {solution }}$, corresponding to mild repulsion between the OZPN molecules in the solution. The likely moderating force is the hydrophobic attraction induced by the structured water, indicated by the $\Delta S_{\text {cryst }}^{o}$ datum for this solvent in Figure 7a. As the structured water molecules are stripped at $C_{E t O H}$ equal or greater than $30 \%, H_{\text {solution }}$, Scheme 2 , suggests stronger intermolecular repulsion that may be due to the hydration shells of the polar nitrogen groups of OZPN, Scheme 1a; note that in contrast to nonpolar moieties, water structured at polar groups induces repulsion due to its strong association with the solute. ${ }^{74,85}$ The attenuated repulsion at higher $C_{E t O H}$ may be due to the lower dielectric constant of these solutions, conducive of stronger van der Waals attraction. ${ }^{86} \Delta G_{\text {cryst }}^{o}$ is a convolution of $\Delta H_{\text {cryst }}^{o}$ and $\Delta S_{\text {cryst }}^{o}$.

Solution thermodynamics dictates the cluster volume fraction. To understand the non-monotonic dependence of $\phi$ on the EtOH content, Figure 4b, we note that direct application of the relation between $\phi$ and $\Delta G_{c l}$, derived above, is not feasible since data on $\Delta G_{c l}$ are unavailable. We use the suggestions for $H_{\text {solution }}$ in solvents of different composition, Scheme 2, as an indicator for the intermolecular interactions between the solute molecules. The variations of $H_{\text {solution }}$ parallel those of $\phi$. This synchronous behavior suggests that stronger repulsion between the solute OZPN molecules, indicated by higher $H_{\text {solution }}$, drives the fraction $v$ and the correlated volume $\phi$. In agreement with the transient dimer model of cluster formation, the cluster population volume and the fraction of solute captures in the clusters are determined by the solution thermodynamics. 
The dimer identity is the final step in the application of the transient dimer model to the OZPN-rich clusters. The known experimental crystal structures of neutral OZPN are comprised of the so-called $\mathrm{SC}_{0}$ dimer, a centrosymmetric motif depicted in Scheme $1 \mathrm{~b} .{ }^{15}{ }^{14,17}$ We put forth that the transient dimer may be akin to that structure. Future work to test this hypothesis is under way.

The finding of the thermodynamic control of the cluster phase volume and the suggested dimer identity imply a thermodynamic and a structural pathways to control the cluster population and hence the nucleation and polymorph transformation pathways directed by the clusters, as seen by Warzecha et al. ${ }^{20}$ The thermodynamic parameters of the solution may be modified by low-concentration additives that act on the intermolecular interactions. The dimer may be suppressed by a subtle structural modification of the molecule, compatible with its biological activity.

\section{Conclusions}

We demonstrate that solutions of the antipsychotic drug OZPN in purely aqueous and mixed EtOH/aqueous solvents exhibit solute-rich clusters of radius $R$ ca. $35 \mathrm{~nm}$ that occupy about $10^{-8}-10^{-7}$ of the solution volume and capture ca. $10^{-7}-10^{-5}$ of the OZPN in the solution. The clusters are disordered and likely liquid and do not represent any of the known OZPN condensed phases. The clusters are of interest since previous work suggests that they may be crucial sites for the nucleation of emerging crystal forms in the course of OZPN polymorph transitions.

We find that the cluster radius $R$ is steady in time and independent of the OZPN concentration and the solvent composition. On the other hand, the volume of the cluster 
population $\phi$, related to the fraction of OZPN molecules captured in the clusters $v$, is a sensitive function of both parameters. We demonstrate that $\phi$ and $v$ are dictated by the thermodynamics of the OZPN solution. The steady mesoscopic $R$, decoupled from the volume of the clusters phase, contradicts predictions of classical theories of phase transformation and recent aggregation models. These discrepancies suggests that the OZPN-rich clusters represent a unique condensed phase. We demonstrate that the unusual cluster behaviors are consistent with the predictions of a model that assumes the formation of OZPN dimers and their decay upon exiting the clusters. We propose that a transient dimer, akin to the centrosymmetric motif present in most of the 60 known OZPN crystal structures, may be the oligomer underlying cluster formation.

Our findings suggest that the cluster population and the nucleation and polymorph transformation pathways, mediated by the clusters, can be controlled by modifying the thermodynamic parameters of the solution and suppressing the formation of dimers or other oligomers.

\section{ASSOCIATED CONTENT}

\section{Supporting Information}

The Supporting Information is available free of charge on the ACS Publications website.

Figures $\mathrm{S} 1, \mathrm{~S} 2$, and $\mathrm{S} 3$ and Tables $\mathrm{S} 1$ and $\mathrm{S} 2$

\section{AUTHOR INFORMATION}

\section{Corresponding Authors}

*vekilov@uh.edu

*alastair.florence@strath.ac.uk 
ORCID

Monika Warzecha: 0000-0001-6166-1089

Alastair J. Florence: 0000-0002-1230-7427

Peter G. Vekilov: 0000-0002-3424-8720

Notes

The authors declare no competing financial interest.

\section{ACKNOWLEDGEMENTS}

We thank Maria Vorontsova, Michael Byington, and Megan Ketchum for help with the experiment techniques and data processing and the EPSRC Centre for Continuous Manufacturing and Crystallisation (CMAC) for access to equipment. This work is supported by grants from EPRSC (EP/K039229/1), NASA (NNX14AD68G and NNX14AE79G), and NSF (MCB-1518204).

\section{REFERENCES}

1. Wöhler; Liebig, Untersuchungen über das Radikal der Benzoesäure. Annalen der Pharmacie 1832, 3 (3), 249-282.

2. Thun, J.; Seyfarth, L.; Senker, J.; Dinnebier, R. E.; Breu, J., Polymorphism in Benzamide: Solving a 175-Year-Old Riddle. Angewandte Chemie International Edition 2007, 46 (35), 6729-6731.

3. Carletta, A.; Meinguet, C.; Wouters, J.; Tilborg, A., Solid-State Investigation of Polymorphism and Tautomerism of Phenylthiazole-thione: A Combined Crystallographic, Calorimetric, and Theoretical Survey. Crystal Growth \& Design 2015, 15 (5), 2461-2473.

4. Johansson, K. E.; van de Streek, J., Revision of the Crystal Structure of the First Molecular Polymorph in History. Crystal Growth \& Design 2016, 16 (3), 1366-1370.

5. Ostwald, W., Studien über die Bildung und Umwandlung fester Körper:. Z. Phys. Chem. 1897, 22, 289-330.

6. Stranski, I.; Totomanov., D., Rate of formation of (crystal) nuclei and the Ostwald step rule. $Z$. phys. Chem. 1933, A163 399-408.

7. Threlfall, T., Structural and Thermodynamic Explanations of Ostwald's Rule. Organic Process Research \& Development 2003, 7 (6), 1017-1027.

8. Bernstein, J., Polymorphism in Molecular Crystals. Oxford University Press: Oxford, 2007. 
9. Blagden, N.; Davey, R. J., Polymorph Selection: Challenges for the Future? Crystal Growth \& Design 2003, 3 (6), 873-885.

10. Müller, M.; Meier, U.; Wieckhusen, D.; Beck, R.; Pfeffer-Hennig, S.; Schneeberger, R., Process Development Strategy to Ascertain Reproducible API Polymorph Manufacture. Crystal Growth \& Design 2006, 6 (4), 946-954.

11. Kirwan, D. J.; Orella, C. J., Crystallization in the pharmaceutical and bioprocessing industries. In Handbook of Industrial Crystallization, Myerson, A. S., Ed. Butterworth-Heinemann: 2002.

12. Chemburkar, S. R.; Bauer, J.; Deming, K.; Spiwek, H.; Patel, K.; Morris, J.; Henry, R.; Spanton, S.; Dziki, W.; Porter, W.; Quick, J.; Bauer, P.; Donaubauer, J.; Narayanan, B. A.; Soldani, M.; Riley, D.; McFarland, K., Dealing with the Impact of Ritonavir Polymorphs on the Late Stages of Bulk Drug Process Development. Organic Process Research \& Development 2000, 4 (5), 413-417.

13. Cherukuvada, S.; Babu, N. J.; Nangia, A., Nitrofurantoin-p-aminobenzoic acid cocrystal: Hydration stability and dissolution rate studies. Journal of Pharmaceutical Sciences 2011, 100 (8), 32333244.

14. Reutzel-Edens, S. M.; Bush, J. K.; Magee, P. A.; Stephenson, G. A.; Byrn, S. R., Anhydrates and Hydrates of Olanzapine: Crystallization, Solid-State Characterization, and Structural Relationships. Crystal Growth \& Design 2003, 3 (6), 897-907.

15. Bhardwaj, R. M.; Price, L. S.; Price, S. L.; Reutzel-Edens, S. M.; Miller, G. J.; Oswald, I. D. H.; Johnston, B. F.; Florence, A. J., Exploring the Experimental and Computed Crystal Energy Landscape of Olanzapine. Crystal Growth \& Design 2013, 13 (4), 1602-1617.

16. Fulton, B.; Goa, K. L., Olanzapine. Drugs 1997, 53 (2), 281-298.

17. Paisana, M. C.; Wahl, M. A.; Pinto, J. F., Role of moisture on the physical stability of polymorphic olanzapine. Int J Pharm 2016, 509 (1-2), 135-148.

18. Sarmah, K. K.; Sarma, A.; Roy, K.; Rao, D. R.; Thakuria, R., Olanzapine Salts and Diversity in Molecular Packing. Crystal Growth \& Design 2016, 16 (2), 1047-1055.

19. Thakuria, R.; Nangia, A., Olanzapinium Salts, Isostructural Solvates, and Their Physicochemical Properties. Crystal Growth \& Design 2013, 13 (8), 3672-3680.

20. Warzecha, M.; Guo, R.; Bhardwaj, R. M.; Reutzel-Edens, S. M.; Price, S. L.; Lamprou, D.; Florence, A. J., Direct observation of templated two-step nucleation mechanism during olanzapine hydration. Crystal Growth \& Design 2017, submitted.

21. Koster, R. S.; Fang, C.; van Blaaderen, A.; Dijkstra, M.; van Huis, M. A., Acetate ligands determine the crystal structure of CdSe nanoplatelets - a density functional theory study. Physical Chemistry Chemical Physics 2016, 18 (32), 22021-22024.

22. Volmer, M., Kinetik der Phasenbildung. Steinkopff: Dresden, 1939; p 208.

23. Volmer, M.; Schultze, W., Kondensation an Kristallen. Z. phys. Chem. 1931, 156A (1), 1-22.

24. Neilsen, A. E., Nucleation in aqueous solutions. In Crystal Growth, Peiser, S., Ed. Pergamon: Oxford, 1967; pp 419-426.

25. Walton, A. G., Nucleation in liquids and solutions. In Nucleation, Zettlemoyer, A. C., Ed. Marcel Dekker: New York, 1969; pp 225-307.

26. Vekilov, P. G., Dense liquid precursor for the nucleation of ordered solid phases from solution. Crystal Growth and Design 2004, 4, 671-685.

27. Galkin, O.; Chen, K.; Nagel, R. L.; Hirsch, R. E.; Vekilov, P. G., Liquid-liquid separation in solutions of normal and sickle cell hemoglobin. Proc. Natl. Acad. Sci. USA 2002, 99, 8479-8483.

28. Garetz, B.; Matic, J.; Myerson, A., Polarization switching of crystal structure in the nonphotochemical light-induced nucleation of supersaturated aqueous glycine solutions. Phys. Rev. Lett. 2002, 89, 175501.

29. Erdemir, D.; Lee, A. Y.; Myerson, A. S., Nucleation of Crystals from Solution: Classical and TwoStep Models. Accounts Chem. Res. 2009, 42 (5), 621-629. 
30. Vorontsova, M. A.; Maes, D.; Vekilov, P. G., Recent advances in the understanding of two-step nucleation of protein crystals. Faraday Discussions 2015, 179 (0), 27-40.

31. Vekilov, P. G., Nucleation. Crystal Growth \& Design 2010, 10 (12), 5007-5019.

32. Yamazaki, T.; Kimura, Y.; Vekilov, P. G.; Furukawa, E.; Shirai, M.; Matsumoto, H.; Van Driessche, A. E. S.; Tsukamoto, K., Two types of amorphous protein particles facilitate crystal nucleation. Proceedings of the National Academy of Sciences 2017, 114, 2154-2159.

33. Auer, S.; Ricchiuto, P.; Kashchiev, D., Two-step nucleation of amyloid fibrils: omnipresent or not? Journal of Molecular Biology 2012, 422 (5), 723-730.

34. Sauter, A.; Roosen-Runge, F.; Zhang, F.; Lotze, G.; Feoktystov, A.; Jacobs, R. M. J.; Schreiber, F., On the question of two-step nucleation in protein crystallization. Faraday Discussions 2015, 179 (0), 41-

58.

35. Schubert, R.; Meyer, A.; Baitan, D.; Dierks, K.; Perbandt, M.; Betzel, C., Real-Time Observation of Protein Dense Liquid Cluster Evolution during Nucleation in Protein Crystallization. Crystal Growth \& Design 2017, 17 (3), 954-958.

36. Saric, A.; Chebaro, Y. C.; Knowles, T. P. J.; Frenkel, D., Crucial role of nonspecific interactions in amyloid nucleation. Proceedings of the National Academy of Sciences 2014, 111 (50), 17869-17874.

37. Savage, J. R.; Dinsmore, A. D., Experimental Evidence for Two-Step Nucleation in Colloidal Crystallization. Physical Review Letters 2009, 102 (19), 198302.

38. Zhang, T. H.; Liu, X. Y., Multistep crystal nucleation: A kinetic study based on colloidal crystallization. Journal of Physical Chemistry B 2007, 111 (50), 14001-14005.

39. Likhatski, M.; Karacharov, A.; Kondrasenko, A.; Mikhlin, Y., On a role of liquid intermediates in nucleation of gold sulfide nanoparticles in aqueous media. Faraday Discussions 2015, 179 (0), 235-245.

40. Gebauer, D.; Kellermeier, M.; Gale, J. D.; Bergstrom, L.; Colfen, H., Pre-nucleation clusters as solute precursors in crystallisation. Chem Soc Rev 2014, 43, 2348-2371.

41. De Yoreo, J., Crystal nucleation: More than one pathway. Nat Mater 2013, 12 (4), 284-285.

42. Dey, A.; Bomans, P. H. H.; Müller, F. A.; Will, J.; Frederik, P. M.; de With, G.; Sommerdijk, N. A. J. M., The role of prenucleation clusters in surface-induced calcium phosphate crystallization. Nat Mater 2010, 9 (12), 1010-1014.

43. Aber, J. E.; Arnold, S.; Garetz, B. A., Strong dc Electric Field Applied to Supersaturated Aqueous Glycine Solution Induces Nucleation of the Polymorph. Phys. Rev. Lett. 2005, 94, 145503.

44. Harano, K.; Homma, T.; Niimi, Y.; Koshino, M.; Suenaga, K.; Leibler, L.; Nakamura, E., Heterogeneous nucleation of organic crystals mediated by single-molecule templates. Nature Materials 2012, 11, 877-881.

45. Bonnett, P. E.; Carpenter, K. J.; Dawson, S.; Davey, R. J., Solution crystallization via a submerged liquid-liquid phase boundary: oiling out. Chem. Commun. 2003, 698-699.

46. Hutchens, S. B.; Wang, Z.-G., Metastable cluster intermediates in the condensation of charged macromolecule solutions. J. Chem. Phys. 2007 127, 084912

47. Johnston, K. P.; Maynard, J. A.; Truskett, T. M.; Borwankar, A. U.; Miller, M. A.; Wilson, B. K.; Dinin, A. K.; Khan, T. A.; Kaczorowski, K. J., Concentrated Dispersions of Equilibrium Protein Nanoclusters That Reversibly Dissociate into Active Monomers. ACS Nano 2012, 6 (2), 1357-1369.

48. Pan, W.; Vekilov, P. G.; Lubchenko, V., The origin of anomalous mesoscopic phases in protein solutions. J. Phys. Chem. B 2010, 114 7620-7630.

49. Maes, D.; Vorontsova, M. A.; Potenza, M. A. C.; Sanvito, T.; Sleutel, M.; Giglio, M.; Vekilov, P. G., Do protein crystals nucleate within dense liquid clusters? Acta Crystallographica Section F 2015, 71 (7), 815-822.

50. Li, Y.; Lubchenko, V.; Vekilov, P. G., The Use of Dynamic Light Scattering and Brownian Microscopy to Characterize Protein Aggregation. Rev. Sci. Instrum. 2011, 82, 053106 
51. Filipe, V.; Hawe, A.; Jiskoot, W., Critical Evaluation of Nanoparticle Tracking Analysis (NTA) by NanoSight for the Measurement of Nanoparticles and Protein Aggregates. Pharmaceutical Research 2010, 27 (5), 796-810.

52. Vorontsova, M. A.; Chan, H. Y.; Lubchenko, V.; Vekilov, P G., Lack of Dependence of the Sizes of the Mesoscopic Protein Clusters on Electrostatics. Biophysical Journal 2015, 109 (9), 1959-1968.

53. Vorontsova, M. A.; Vekilov, P. G.; Maes, D., Characterization of the diffusive dynamics of particles with time-dependent asymmetric microscopy intensity profiles. Soft Matter 2016, 12 (33), 6926-6936.

54. Wawrzycka-Gorczyca, I.; Borowski, P.; Osypiuk-Tomasik, J.; Mazur, L.; Koziol, A., Crystal structure of olanzapine and its solvates. Part 3. Two and three-component solvates with water, ethanol, butan-2-ol and dichloromethane. 2007; Vol. 830, p 188-197.

55. Sedlák, M.; Rak, D., Large-Scale Inhomogeneities in Solutions of Low Molar Mass Compounds and Mixtures of Liquids: Supramolecular Structures or Nanobubbles? The Journal of Physical Chemistry $B$ 2013, 117 (8), 2495-2504.

56. Chaikin, P. M.; Lubensky, T. C., Principles of condensed matter physics. Cambridge Unversity Press: Cambridge, 1995.

57. Stradner, A.; Sedgwick, H.; Cardinaux, F.; Poon, W. C. K.; Egelhaaf, S. U.; Schurtenberger, P., Equilibrium cluster formation in concentrated protein solutions and colloids. Nature 2004, 432 (7016), 492-495.

58. Porcar, L.; Falus, P.; Chen, W.-R.; Faraone, A.; Fratini, E.; Hong, K.; Baglioni, P.; Liu, Y., Formation of the Dynamic Clusters in Concentrated Lysozyme Protein Solutions. The Journal of Physical Chemistry Letters 2009, 1 (1), 126-129.

59. Erlkamp, M.; Grobelny, S.; Faraone, A.; Czeslik, C.; Winter, R., Solvent Effects on the Dynamics of Amyloidogenic Insulin Revealed by Neutron Spin Echo Spectroscopy. The Journal of Physical Chemistry $B$ 2014, 118 (12), 3310-3316.

60. Borwankar, A. U.; Dinin, A. K.; Laber, J. R.; Twu, A.; Wilson, B. K.; Maynard, J. A.; Truskett, T. M.; Johnston, K. P., Tunable equilibrium nanocluster dispersions at high protein concentrations. Soft Matter 2013, 9 (6), 1766-1771.

61. Soraruf, D.; Roosen-Runge, F.; Grimaldo, M.; Zanini, F.; Schweins, R.; Seydel, T.; Zhang, F.; Roth, R.; Oettel, M.; Schreiber, F., Protein cluster formation in aqueous solution in the presence of multivalent metal ions - a light scattering study. Soft Matter 2014, 10 (6), 894-902.

62. Yearley, Eric J.; Godfrin, Paul D.; Perevozchikova, T.; Zhang, H.; Falus, P.; Porcar, L.; Nagao, M.; Curtis, Joseph E.; Gawande, P.; Taing, R.; Zarraga, Isidro E.; Wagner, Norman J.; Liu, Y., Observation of Small Cluster Formation in Concentrated Monoclonal Antibody Solutions and Its Implications to Solution Viscosity. Biophysical Journal 2014, 106 (8), 1763-1770.

63. Cardinaux, F.; Stradner, A.; Schurtenberger, P.; Sciortino, F.; Zaccarelli, E., Modeling equilibrium clusters in lysozyme solutions. EPL (Europhysics Letters) 2007, 77 (4), 48004.

64. Gliko, O.; Neumaier, N.; Pan, W.; Haase, I.; Fischer, M.; Bacher, A.; Weinkauf, S.; Vekilov, P. G., A metastable prerequisite for the growth of lumazine synthase crystals. J. Amer. Chem. Soc. 2005, 127, 3433-3438.

65. Li, Y.; Lubchenko, V.; Vorontsova, M. A.; Filobelo, L.; Vekilov, P. G., Ostwald-Like Ripening of the Anomalous Mesoscopic Clusters in Protein Solutions. The Journal of Physical Chemistry B 2012, 116 (35), 10657-10664.

66. Gliko, O.; Pan, W.; Katsonis, P.; Neumaier, N.; Galkin, O.; Weinkauf, S.; Vekilov, P. G., Metastable liquid clusters in super- and undersaturated protein solutions. J. Phys. Chem. B 2007, 111 (12), 31063114. 
67. Sleutel, M.; Van Driessche, A. E., Role of clusters in nonclassical nucleation and growth of protein crystals. Proceedings of the National Academy of Sciences of the United States of America 2014, 111 (5), E546-53.

68. Uzunova, V. V.; Pan, W.; Galkin, O.; Vekilov, P. G., Free heme and the polymerization of sickle cell hemoglobin. Biophys. J. 2010, 99 (6), 1976-1985.

69. Lutsko, J. F., Mechanism for the stabilization of protein clusters above the solubility curve: the role of non-ideal chemical reactions. J. Phys.-Condes. Matter 2016, 28 (24).

70. Lutsko, J. F.; Nicolis, G., Mechanism for the stabilization of protein clusters above the solubility curve. Soft Matter 2015, 12, 93-98.

71. Byington, M. C.; Safari, M. S.; Conrad, J. C.; Vekilov, P. G., Shear flow suppresses the volume of the nucleation precursor clusters in lysozyme solutions. J. Cryst. Growth 2017, 468, 493-501.

72. Olafson, K. N.; Ketchum, M. A.; Rimer, J. D.; Vekilov, P. G., Mechanisms of hematin crystallization and inhibition by the antimalarial drug chloroquine. Proceedings of the National Academy of Sciences 2015, 112 (16), 4946-4951.

73. Derewenda, Z. S.; Vekilov, P. G., Entropy and surface engineering in protein crystallization. Acta Crystallogr. Section D 2006, 62, 116-124.

74. Leckband, D.; Israelachvili, J., Intermolecular forces in biology. Quart. Rev. Biophysics 2001, 34, 105-267.

75. Raviv, U.; Klien, J., Fluiidity of bound hydration layers. Science 2002, 297, 1540-1543.

76. Ball, P., Water as an Active Constituent in Cell Biology. Chem. Rev. 2008, 108 (1), 74-108.

77. Pal, S. K.; Zewail, A. H., Dynamics of Water in Biological Recognition. Chem. Rev. 2004, 104 (4), $2099-2124$.

78. Tanford, C., The hydrophobic effect: formation of micelles and biological membranes. John Wiley \& Sons: New York, 1980.

79. Eisenberg, D.; Kauzmann, W., The structure and properties of water. University Press: Oxford, 1969.

80. Eisenberg, D.; Crothers, D., Physical Chemistry with Applications to Life Sciences. The Benjamin/Cummins: Menlo Park, 1979.

81. Dunitz, J. D., The entropic cost of bound water in crystals and biomolecules. Science 1994, 264, 670-670.

82. Fersht, A., Structure and mechanism in protein science. W.H. Freeman: New York, 1999.

83. Haynes, W. M., CRC Handbook of Chemistry and Physics (92nd ed.). CRC Press: Boca Raton, FL, $2011 ;$ p 3.246.

84. Dill, K.; Bromberg, S., Molecular Driving Forces: Statistical Thermodynamics in Chemistry and Biology. Garland Science: New York, 2003.

85. Petsev, D. N.; Vekilov, P. G., Evidence for Non-DLVO Hydration Interactions in Solutions of the Protein Apoferritin. Phys. Rev. Lett. 2000, 84, 1339-1342.

86. Berry, P. S.; Rice, S. A.; Ross, J., Physical Chemistry. Second ed.; Oxford University Press: New York, 2000. 